\title{
Pulsational behaviour of the HADS RY Lep
}

\author{
E. Rodríguez ${ }^{1}$, C.D. Laney ${ }^{2}$, P.J. Amado ${ }^{1}$, M.J. López-González ${ }^{1}$, M. Joner ${ }^{3}$ \\ 1 Instituto de Astrofísica de Andalucía, CSIC, P.O. Box 3004, E-18080 Granada, Spain \\ 2 South African Astronomical Observatory, P.O. Box 9, Observatory 7935, Cape Town, South Africa \\ ${ }^{3}$ Department of Physics and Astronomy, Brigham Young University, Provo, Utah 84602, USA
}

RY Lep is a long-period High Amplitude $\delta$ Sct-type pulsator $\left(\mathrm{V}=8 .^{m} 21, \Delta \mathrm{V}=0 .{ }^{m} 35\right)$ with a main period of $0 .{ }^{d} 2254$ (Diethelm 1985). This star was assumed to be monoperiodic with stable light curves for a long time. However, some disturbances have recently been discovered by Laney et al. (2002). New observations were collected at Sierra Nevada Observatory (SNO), Spain, with simultaneous uvby photometry, from 1998 to 2002, but only the two data sets collected in 1998 and 2002 are good enough to perform individual Fourier analyses. Additional VJHK data were collected at South African Astronomical Observatory (SAAO), South Africa, during the same epoch.

Besides the main periodicity at $f_{1}=4.4416 \mathrm{~cd}^{-1}$, the existence of a secondary frequency at $\mathrm{f}_{2}=6.60 \mathrm{~cd}^{-1}$ was confirmed when Fourier analyses were performed for each of the reliable data sets available: our 1998 and 2002 SNO data sets, those of Hipparcos satellite (ESA 1997) and those of Diethelm (1985). Moreover, in some sets it seems that combinations of $f_{1}$ and $f_{2}$ are also present $\left(f_{2}+f_{1}\right.$ in $1998 ; f_{2}+f_{1}$ and $f_{2}-f_{1}$ in the Hipparcos data). This corroborates the existence and pulsational nature of $f_{2}$ together with the observed amplitude ratios and phase shifts between different filters obtained for $\mathrm{f}_{2}$ and $u v b y$ and BV photometry. Amplitude variations from season to season are also evident for $f_{2}$, however there are no significant variations for $f_{1}$. On the other hand, the derived phase shifts in uvby and BV photometry suggest radial pulsation for $f_{1}$ and nonradial for $f_{2}$. In the case of $f_{1}$, it also agrees with the results of Rodríguez et al. (1995). Moreover, a $\mathrm{Q}$ value of $0 .{ }^{d} 033$ is found for $\mathrm{f}_{1}$, which is indication of pulsation in the fundamental mode. This suggests that $f_{2}$ corresponds to a nonradial $\mathrm{p}_{2}$ mode.

The analysis of the VJHK data collected at SAAO confirmed the existence of $f_{2}$ and the nature of the modes. Although the expected pulsational amplitude in the infrared filters is very small (about $30 \%$ and $20 \%$ in $\mathrm{J}$ and $\mathrm{K}$ relative to the amplitude observed in the $v$ filter (Balona \& Evers 1999)), $\mathrm{f}_{2}$ was detected in the J filter of the data set collected during the year 2000. As compared with the vby filters, the phase shifts confirm the radial nature of $f_{1}$ and the nonradial one for $f_{2}$ with an angular quantum number of $\mathrm{I}=2$.

In summary, the pulsational behaviour of RY Lep can be well described by a main frequency $f_{1}$, stable in amplitude and being the fundamental radial mode, and a secondary frequency $\mathrm{f}_{2}$, variable in amplitude from season to season and corresponding to nonradial pulsation with $\mathrm{p}_{2}$ and $\mathrm{I}=2$. Moreover, binarity is also suggested from our study when an $\mathrm{O}-\mathrm{C}$ analysis is performed with the derived times of light maximum.

\section{References}

Balona L.A., Evers E.A. 1999, MNRAS 302, 349

Diethelm R. 1985, A\&A 149, 465

ESA 1997, The Hipparcos and Tycho Catalogues, ESA SP-1200

Laney C.D., Joner M., Schwendiman L. 2002, PASPC 256, 173

Rodríguez E., Rolland A., Costa V., Martín S. 1995, MNRAS 277, 965 